\title{
Elaboración de Curvas de Destilación de Hidrocarburos Líquidos Pertenecientes a la Sub-Cuenca Neiva: Método ASTM D86-04
}

\section{Elaboration of Distillation Curves of Liquid Hydrocarbons Belonging to the Neiva Sub-Basin: Method ASTM D86-04}

\author{
Haydee Morales', Shirley Zamara Vargas ${ }^{2}$ y José Avelino Olaya ${ }^{3}$
}

\section{Resumen}

En este articulo se presenta las curvas de destilación elaboradas del análisis a 10 muestras de crudos pertenecientes a la Sub-Cuenca Neiva por medio de una investigación experimental. Esta experiencia se llevó a cabo en un equipo de destilación manual Koehler modelo G-142-K45000 al cual se le realizó el mamual de operaciones conforme al método estandarizado ASTMD86-04. Para lograr la caracterización de los crudos segín su base, los datos obtenidos experimentalmente inicialmente fueron corregidos por presión, pérdidas y por craqueo térmico; este último parámetro según Hadden (1990) (citado por Perry, 1997), posteriormente se utilizan las expresiones propuestas por Watson (1935), Whitson (1980) y el cálculo de la Constante de Gravedad - Viscosidad (VGC) para lograr la caracterización segín su base de las muestras estudiadas; también se presenta un reporte detallado con los resultados obtenidos mediante el análisis de las curvas de destilación de las fracciones C7+ como: Temperatura promedio volumétrica, Temperatura promedio peso, Temperatura promedio cúbica, Temperatura promedio molal, Gravedad específica, Factor de caracterización, Peso molecular y Propiedades seudocríticas, por medio del cual se permite clasificar las muestras de los campos A y B, en base mixta: Nafténica- Parafinica, con influencia predominante nafténica; las muestras de los campos C, D y E, en base mixta: Parafinica- Nafténica, con influencia predominante parafinica y la muestra del campo F: Nafténica- Aromática con influencia predominante nafténica.

Palabras claves: Caracterización de los crudos según su base; Curvas de destilación; Método estandarizado ASTM D86-04.

\begin{abstract}
In this article, it is presented the distillation curves elaborated through the analysis to ten samples of crude oil, belonging to Neiva Sub-Basin through an experimental research. This experience is carried out in a manual distillation equipment Koehler model G-142-K45000 to which the manual operations were performed according to the standardized method ASTM D86-04. To obtain the crudes characterization according to their basis, the data obtained experimentally, are corrected, initially by pressure, losses and by thermal cracking, this last parameter, according to S.T. Hadden(1990) (cited by Perry, 1997), later on the expressions proposed by Watson (1935), Whitson (1980) and the calculation of the gravity constant - viscosity are used to obtain the characterization according to its basin of the studied samples; it is also provided a detailed report with the obtained results by means of the analysis of the distillation curves of the fractions $\mathrm{C} 7+$ as: volumetric average temperature, weight average temperature, cubic average temperature, molar average temperature, specific gravity, characterization factor, molecular weight and pseudo critical properties, by which we would like to classify the samples of the fields A and B, based mixed: naphthenic-paraffinic, naphthenic with predominant influence, and samples of the fields $\mathrm{C}, \mathrm{D}$ and $\mathrm{E}$, based mixed: Paraffin-naphthenic, with predominant influence paraffinic and field sample F: Aromatic with naphthenic-naphthenic predominant influence.
\end{abstract}

Key Words: Characterization of crude oils according to their basis; curves of distillation; standardized method ASTM D8604.

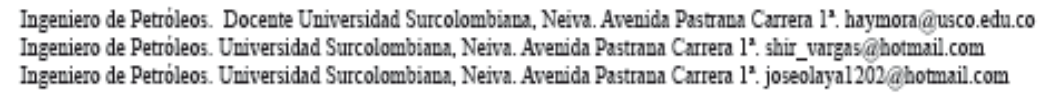




\section{Introducción}

Las propiedades fisicoquímicas de los petróleos varían con su procedencia, incluso si se trata de distintos pozos de un mismo yacimiento y también en los crudos extraidos de las diferentes zonas productoras en el mismo pozo. La bibliografia especializada habla de hidrocarburos de más de 80 átomos de carbono presentes en los petróleos que dan mezclas sumamente complejas (Cerutti, 2002).

La capacidad del petróleo para producir una lista de productos predeterminados se determinará por la aplicación de una serie de métodos analíticos (Speight, 2001) que proporcionan información suficiente para evaluar la calidad potencial del petróleo como materia prima, de acuerdo a las propiedades físicas tales como el punto de ebullición, la densidad relativa y la gravedad API se expresa la calidad del petróleo crudo, dando aproximadamente el carácter general, pero no su composición química. Para su análisis, procesamiento y comercialización se clasifica en varios grupos llamados bases, la cual se determina por medio del tipo estructural y la cantidad relativa de hidrocarburos presentes (Camillo, 1985).

El aislamiento de los compuestos puros de petróleo ha sido una tarea compleja, junto con los constituyentes de las fracciones de mayor peso molecular, y la presencia de compuestos de azufre, oxigeno y nitrógeno; siendo la causa principal de la dificultades encontradas en el momento de producir, transportar, almacenar y refinar, de ahí la necesidad de un conocimiento más exacto de la composición de los crudos y sus productos (Gerald, 2009).

La destilación es el primer procedimiento realizado al petróleo crudo por medio de calor para realizar la separación de sus componentes permitiendo la determinación del rendimiento de los diferentes cortes como gas, nafta, gasolina, etc. Por medio de este procedimiento de laboratorio se obtienen propiedades necesarias para la realización de las curvas de destilación.

A pesar del considerable desarrollo de la termodinámica y de las teorias moleculares, la mayoría de los métodos utilizados hoy en dia son empíricos, y su utilización exige el conocimiento de valores experimentales. Sin embargo, se recurre a métodos predictivos para obtener valores precisos que se necesitan en la industria y que no se pueden obtener experimentalmente a fin de obtener un análisis completo y un aprovechamiento a las curvas de destilación (Rand, 2003).

\section{Metodología}

Esta investigación en primera instancia, puntualiza de forma habitual, los cálculos y ajustes realizados a las pruebas de destilación según el método ASTM D 86-04 para obtener las respectivas curvas de destilación. En segunda instancia, se hace énfasis en tres métodos con los cuáles se caracterizan las muestras de crudo según la naturaleza de hidrocarburos contenidos en estos.

La técnica ASTM D 86-04 fue utilizada al ser un método de estudio sistemático, analítico, descriptivo y sencillo para conseguir el objetivo de la investigación. El procedimiento es barato, rápido de ejecutar, y repetible en un grado razonable de precisión (por lo general de $6^{\circ} \mathrm{F}\left[3,3^{\circ} \mathrm{C}\right]$ ) (ASTM, 2004).

La temperatura y el volumen condensado son tomados simultáneamente, generando como resultado el punto de ebullición como una función del volumen condensado; el resultado del ensayo se calcula y refleja bajo la forma de temperatura de ebullición en función del volumen destilado.

Para el procesamiento de los datos se hizo uso de principios matemáticos y de ingeniería y las ecuaciones que describen y explican el comportamiento de la temperatura de destilación de las fracciones de hidrocarburos $\mathrm{C} 7+$ para generar soluciones analíticas basadas en la aplicación de la técnica ASTM D- 86-04 (ASTM, 2004) y con las cuales es posible especificar los crudos segín su base o naturaleza.

2.1 Corrección de la temperatura y el volumen en función de la presión de la prueba y las pérdidas.

La ecuación (1) se desarrolló durante el curso del trabajo de Sidney Young en separaciones de destilación y mediciones de equilibrio (vapor + líquido), el uso de la constante es una simplificación de la forma original de la ecuación SY (Young, 1922).

$$
T_{760}=T_{p}+0.0009(101.3-P)\left(273-T_{p}\right)
$$

Los datos de volumen de las pruebas de destilación se corrigen por pérdidas, como sigue:

$$
L_{c}=A L+B
$$


Luego hallamos el volumen corregido por pérdidas en la destilación:

$$
V_{c_{i}}=V_{i}+\left(\frac{L_{i}}{L_{T}}\right) * L_{C}
$$

\subsection{Corrección según S.T Hadden.}

Aunque la mayor parte del petróleo crudo puede ser calentado a $600{ }^{\circ} \mathrm{F}\left(316^{\circ} \mathrm{C}\right)$ sin agrietamiento notable, cuando las temperaturas ASTM exceden $475^{\circ} \mathrm{F}\left(246^{\circ} \mathrm{C}\right)$, los gases pueden desplegarse, lo que indica descomposición, que puede causar lecturas bajas del termómetro. En ese caso, se aplica la siguiente ecuación (Penry, 1997):

$$
\Delta T_{\text {Corr }}=10^{-1.587+0.004735 T}
$$

\section{Resultados}

La tabla 1 muestra los datos adquiridos en la destilación ASTM D-86-04 a la muestra A1: En la primera columna el porcentaje de volumen obtenido, en la segunda la corrección del volumen debido a las pérdidas, en la tercera columna la temperatura en ${ }^{\circ} \mathrm{C}$ y ${ }^{\circ} \mathrm{F}$ observada y en la cuarta columna los datos de temperaturas llevados a presión de $760 \mathrm{~mm} \mathrm{Hg}$.

\begin{tabular}{|c|c|c|c|c|c|}
\hline \multirow{3}{*}{$\begin{array}{c}\% \\
\text { Volumen } \\
\text { Recobrado }\end{array}$} & \multirow{3}{*}{$\begin{array}{c}\% \\
\text { Volumen corregido por } \\
\text { pérdidas, V_(c_i ) }\end{array}$} & \multicolumn{4}{|c|}{ Prueba X } \\
\hline & & \multicolumn{2}{|c|}{ Observado } & \multicolumn{2}{|c|}{ Corregido } \\
\hline & & ${ }^{\circ} \mathrm{C}$ & ${ }^{\circ} \mathrm{F}$ & ${ }^{\circ} \mathrm{C}$ & ${ }^{\circ} \mathrm{F}$ \\
\hline 0 & 0.48 & 84 & 183.2 & 85.6 & 186.1 \\
\hline 5 & 5.96 & 131 & 267.8 & 132.8 & 271.1 \\
\hline 10 & 11.44 & 162 & 323.6 & 164.0 & 327.1 \\
\hline 15 & 16.92 & 184 & 363.2 & 186.1 & 366.9 \\
\hline 20 & 22.40 & 207 & 404.6 & 209.2 & 408.5 \\
\hline 30 & 32.88 & 237 & 458.6 & 239.3 & 462.7 \\
\hline 40 & 43.37 & 310 & 590 & 312.6 & 594.7 \\
\hline 50 & 53.85 & 338 & 640.4 & 340.7 & 645.3 \\
\hline 60 & 64.33 & 339 & 642.2 & 341.8 & 647.2 \\
\hline EP & 73.31 & 346 & 654.8 & 348.7 & 659.7 \\
\hline \multicolumn{2}{|l|}{ Recobrado, \% } & \multicolumn{2}{|c|}{68.5} & \multicolumn{2}{|c|}{71.2} \\
\hline \multicolumn{2}{|l|}{ Residuo, \% } & \multicolumn{2}{|c|}{24} & \multicolumn{2}{|c|}{24} \\
\hline \multicolumn{2}{|l|}{ Pérdida, \% } & \multicolumn{2}{|c|}{7.5} & \multicolumn{2}{|c|}{4.8} \\
\hline
\end{tabular}

Tabla 1. Resultado de la destilación ASTM D86-04 de la Muestra A1.

Con los datos de la tabla 1 , se realizan las respectivas curvas ASTM preliminar corrigiendo el volumen por pérdidas.

Con los datos experimentales de la figura 1 se realizó la curva ASTM y se extrapoló para obtener estos valores de temperatura de ebullición en función de la línea de tendencia de la curva. 


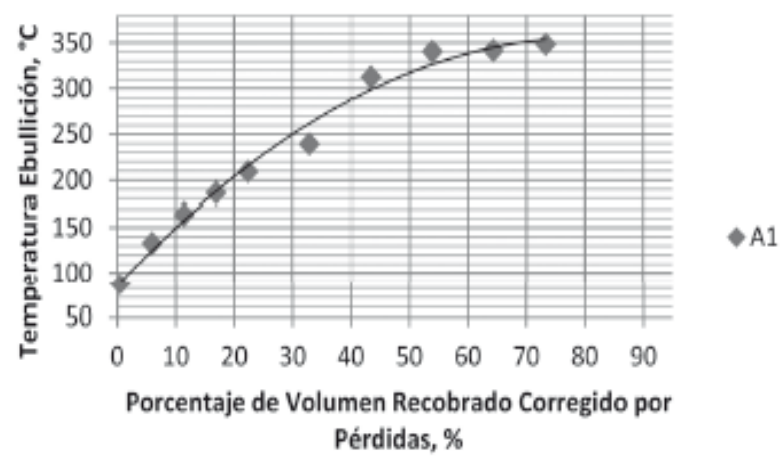

Fig. 1 Curva ASTM preliminar del pozo A1

Los datos de la destilación ASTM D-86-04 se muestran corregidos por presión atmosférica y las temperaturas superiores a $475^{\circ} \mathrm{F}\left(246^{\circ} \mathrm{C}\right)$ se comigen por craqueo segín Hadden (1990) (citado por Perry, 1997), según la ecuación (4), luego los resultados obtenidos se tabulan en la tabla 2.

Tabla 2. Curva ASTM a presión atmosférica.

\begin{tabular}{cc|c}
\hline Muestra & $\begin{array}{c}\text { \% } \\
\text { Recobrado }\end{array}$ & $\begin{array}{c}\text { ASTM D-86-04 } \\
\text { Prueba X, }{ }^{\circ} \mathrm{C}\end{array}$ \\
\hline \multirow{4}{*}{ A1 } & 0 & 84.2 \\
& 10 & 147.1 \\
& 30 & 247.9 \\
& 50 & 315.3 \\
& 70 & 349.2 \\
\hline
\end{tabular}

Para logar una visualización más amplia de los datos plasmados, se procede a realizar las respectivas gráficas donde se muestran las curvas ASTM finales de los campos evaluados (ver figuras 2 a 5).

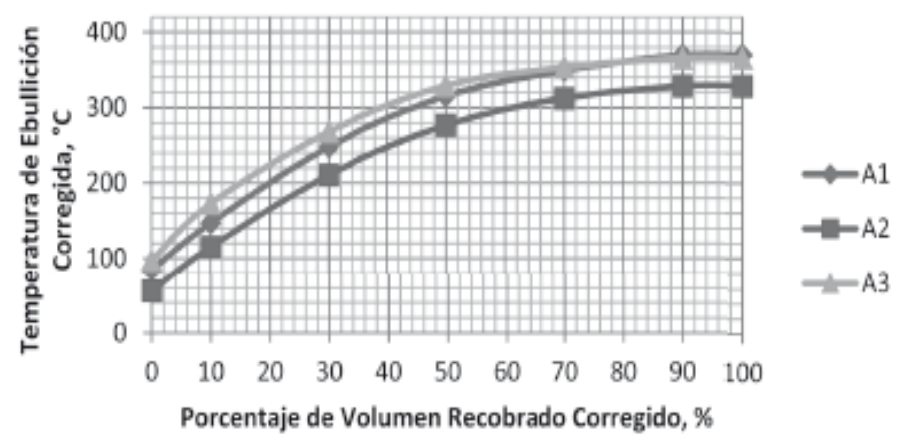

Fig. 2 Curvas de destilación ASTM final, Campo A 


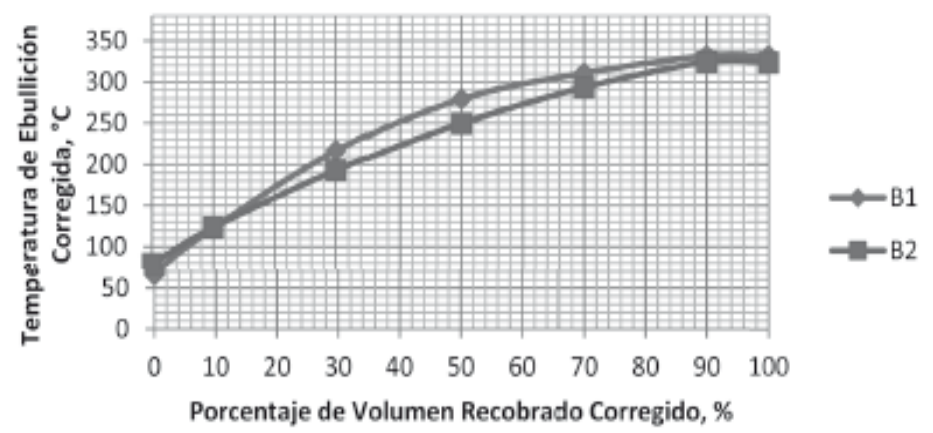

Fig. 3 Curvas de destilación ASTM final, Campo B

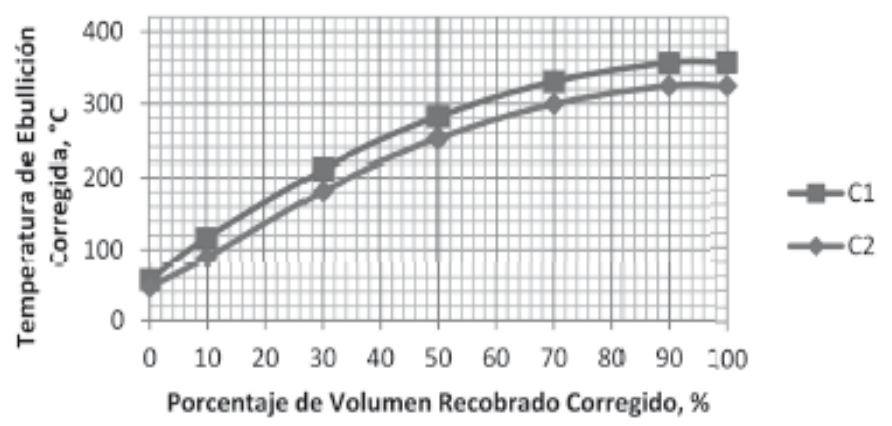

Fig. 4 Curvas de destilación ASTM final, Campo C

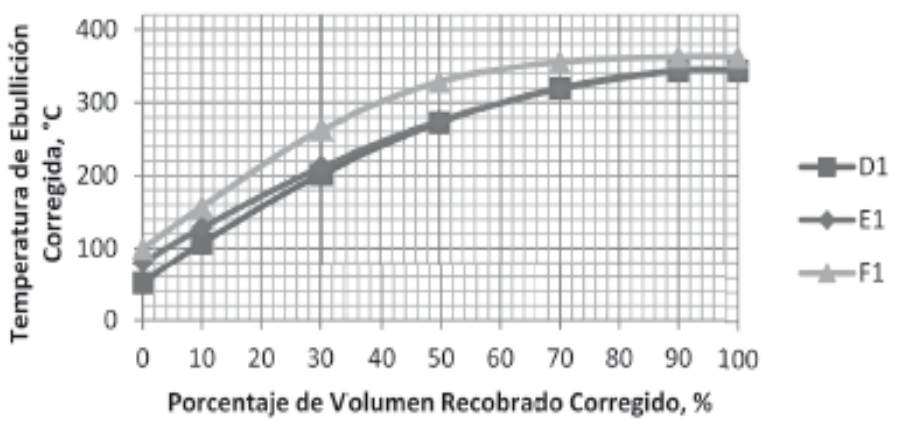

Fig. 5 Curvas de destilación ASTM finales, Campos D, E y F

Factor de caracterización de Watson-Murphy, $\mathrm{K}_{\mathrm{w}}$. Para los hidrocarburos puros que depende únicamente de sus puntos de ebullición (ASTM D86 o ASTM D-1160) y de su densidad (ASTM D-1298) (Cerutti, 2002).

$$
K_{W}=\frac{\left(T_{b}\right)^{1 / 3}}{\gamma}
$$


Corrección generalizada de Whitson. Whitson (1980) sugiere que el factor de Watson puede correlacionarse con el peso molecular (M) y la gravedad específica $(\gamma)$ en la siguiente expresión (Ahmed, 2007):

$$
K_{w} \approx 4.5579\left(\frac{M^{0.15178}}{\gamma^{0.84573}}\right)
$$

Así, la $\mathrm{K}_{\mathrm{w}}$ de los hidrocarburos para las diferentes series químicas tanto para el método de Watson-Murphy como el de Whitson, son los siguientes (Speight, 2002):

- Compuestos parafínicos tienen un $\mathrm{K}_{\mathrm{w}}$ del orden de 12.5 a 13.

- Compuestos nafténicos, del orden de 10.5 a 12.5 .

- Compuestos aromáticos, del orden menor de 10.5

Constante de viscosidad - gravedad (VGC) de crudos. Es una función útil para la caracterización aproximada de las fracciones viscosas del petróleo. Es relativamente insensible al peso molecular y está relacionada con la composición de los fluidos. Este método requiere calcular la viscosidad a $40^{\circ} \mathrm{C}\left(104^{\circ} \mathrm{F}\right)$ y la densidad a $15.55^{\circ} \mathrm{C}\left(60^{\circ} \mathrm{F}\right)$. A partir de estos datos se utiliza la siguiente ecuación para calcular el VGC (ASTM D 250-91, 2005):

$$
V G C=\frac{G-0.0664-0.1156 * \log (V-5.5)}{0.94-0.109 * \log (V-5.5)}
$$

Los valores de VGC de 0,8 equivalen a una muestra parafinica, a medida que aumenta el VGC aumenta el contenido de nafténicos hasta llegar a 0,9 que corresponde a una muestra nafténica. En aumento de VGC después de 0,9 aumenta el contenido de aromáticos hasta llegar a 0,98 que corresponde a una muestra aromática.

La tabla 3 muestra los valores obtenidos a partir de la correlación generalizada de Whitson (1980) para las muestras de los campos en estudio:

\begin{tabular}{|c|c|c|c|c|}
\hline Muestra & $\begin{array}{l}\rho @ 60 \mathrm{~F} \\
(\mathrm{gr} / \mathrm{ml})\end{array}$ & $\begin{array}{c}\text { Factor } \\
\text { Kw }\end{array}$ & $\begin{array}{c}\text { Factor Kw } \\
\text { Whitson }\end{array}$ & VGC \\
\hline Al & 0.9090 & 10.99 & 10.93 & 0.869 \\
\hline A2 & 0.9202 & 10.59 & 10.73 & 0.875 \\
\hline A3 & 0.9299 & 10.81 & 10.97 & 0.888 \\
\hline $\mathrm{Bl}$ & 0.8731 & 11.19 & 11.33 & 0.829 \\
\hline B2 & 0.8851 & 10.92 & 11.07 & 0.847 \\
\hline $\mathrm{Cl}$ & 0.8526 & 11.49 & 11.65 & 0.815 \\
\hline $\mathrm{C} 2$ & 0.8272 & 11.61 & 11.75 & 0.811 \\
\hline Dl & 0.8375 & 11.62 & 11.77 & 0.810 \\
\hline El & 0.8345 & 11.72 & 11.86 & 0.815 \\
\hline F1 & 0.9681 & 10.35 & 10.49 & 0.924 \\
\hline
\end{tabular}

Tabla 3. Factores de caracterización de los crudos por Watson, Whitson y la Constante Viscosidad-Gravedad.

Los valores para los factores de caracterización Kw de Watson (1935) y Whitson (1980), muestran que segin el rango, las muestras: A1, A2, A3, B1 B2, C1, C2, D1 y E1 son base nafténica, mientras la muestra F1 es de base aromática.

Segín los parámetros de la Constante Viscosidad-Gravedad, las muestras A1, A2, A3, son crudos de base Nafténico Parafinica, con influencia nafténica. Las muestras B1, B2, C1, C2, D1 y E1 son muestras de base Parafinico - nafténica, con influencia parafínica. La muestra F1 es de base Nafténica- aromática, con influencia predominante nafténica.

Propiedades Adicionales. La tabla 4 contiene los factores de corrección para evaluar la temperatura promedio en peso (TPW), temperatura promedio medio (TPMe) y la temperatura promedio molal (TPM) leidos de la grafica "caracterizacion del punto de ebullicion de los factores del petroleo" (GPSA, Libros de datos técnicos API) (Parra, 1997). 
Facultad de Ingeniería Universidad Surcolombiana

Tabla 4. Factores de corrección de la TPV para obtener otras temperaturas de ebullición

\begin{tabular}{cccccc}
\hline Muestra & $\begin{array}{c}\text { Temperatura } \\
\text { Promedio Volumétrica }\end{array}$ & Pendiente & \multicolumn{3}{c}{ Factor de Corrección } \\
\hline & ${ }^{\circ}$ F & S & FC (TPW) & FC (TPMe) & FC (TPM) \\
\hline A1 & 546.5 & 5.41 & 19 & -42 & -72 \\
\hline A2 & 479.7 & 5.21 & 14 & -37 & -61 \\
\hline A3 & 568 & 4.74 & 12 & -36 & -58 \\
\hline B1 & 485.7 & 5.1 & 13 & -41 & -66 \\
\hline B2 & 457.9 & 4.92 & 14 & -39 & -64 \\
\hline C1 & 498.8 & 5.81 & 16 & -51 & -85 \\
\hline C2 & 444.3 & 5.72 & 17 & -51 & -83 \\
\hline D1 & 480.3 & 5.73 & 17 & -50 & -82 \\
\hline E1 & 491.5 & 5.24 & 13 & -39 & -65 \\
F1 & 559.9 & 5.03 & 12 & -37 & -62 \\
\hline
\end{tabular}

Las siguientes ecuaciones fueron utilizadas para completar el reporte de la curva de destilación ASTM D-86-04, mostrado en la tabla 5:

- Temperaturas promedios

$$
\begin{aligned}
& T P W=T P V+F_{-} C \\
& T P M=T P V+F_{C} C
\end{aligned}
$$

Dónde:

TPV: Para las ecuaciones 8 a 10 se encuentra en la columna 2 de la tabla 4 .

Fc: Factor de corrección diferente para cada temperatura, ver tabla 4.

- Condiciones Seudocríticas

$$
\begin{array}{r}
s P_{C}=3.12281\left(10^{9}\right) * T P M e^{-2.3125} * \gamma^{2.3021} \\
s T_{C}=24.2187 * T P M e^{0.58848} * \gamma^{0.3596}
\end{array}
$$

\begin{tabular}{|c|c|c|c|c|c|c|c|c|c|c|}
\hline TABLADE RESULTADOS & $\mathrm{Al}$ & $\overline{\mathrm{A} 2}$ & $\overline{\mathrm{A} 3}$ & $\mathrm{Bl}$ & B2 & $\mathrm{Cl}$ & $\mathrm{C} 2$ & Dl & El & F1 \\
\hline $\begin{array}{l}\text { Temperatura Promedio } \\
\text { Volumétrica (TPV), }{ }^{\circ} \mathrm{F} \text {. }\end{array}$ & 546.5 & 479.7 & 568 & 485.7 & 457.9 & 498.8 & 444.3 & 480.3 & 491.5 & 559.9 \\
\hline $\begin{array}{l}\text { Temperatura Promedio Peso } \\
\text { (TPW), }{ }^{\circ} \mathrm{F} \text {. }\end{array}$ & 565.5 & 493.7 & 580 & 498.7 & 471.9 & 514.8 & 461.3 & 497.3 & 504.5 & 571.9 \\
\hline $\begin{array}{l}\text { Temperatura Promedio Cubico } \\
\text { (TPC), }{ }^{\circ} \mathrm{F} \text {. }\end{array}$ & 535.2 & 464.5 & 556.3 & 471 & 443.8 & 480.5 & 425.6 & 462.2 & 476.3 & 546.7 \\
\hline $\begin{array}{l}\text { Temperatura Promedio Medio } \\
\text { (TPMe), }{ }^{\circ} \mathrm{F} \text {. }\end{array}$ & 504.5 & 442.7 & 532 & 444.7 & 418.9 & 447.8 & 393.3 & 430.3 & 452.5 & 522.9 \\
\hline $\begin{array}{l}\text { Temperatura Promedio Molal } \\
\text { (TPM), }{ }^{\circ} \mathrm{F} \text {. }\end{array}$ & 474.5 & 418.7 & 510 & 419.7 & 393.9 & 413.8 & 361.3 & 398.3 & 426.5 & 497.9 \\
\hline Gravedad Especifica ( ) & 0.9088 & 0.92 & 0.9297 & 0.8729 & 0.8849 & 0.8524 & 0.827 & 0.8373 & 0.8343 & 0.9679 \\
\hline Factor de Caracterización (Koup) & 10.99 & 10.59 & 10.81 & 11.19 & 10.92 & 11.49 & 11.61 & 11.62 & 11.72 & 10.35 \\
\hline Temperatura Seudocrítica $(\mathrm{sTc}),{ }^{\circ} \mathrm{F}$. & 875 & 830 & 908 & 807 & 792 & 799 & 741 & 776 & 793 & 921 \\
\hline Presión Seudocrítica (sPc), Psia. & 820 & 968 & 786 & 914 & 990 & 885 & 991 & 909 & 856 & 83622 \\
\hline
\end{tabular}

Tabla 5. Reporte del método ASTM D-86-04 


\section{Conclusiones}

Se logró caracterizar todas las muestras del presente estudio, a través del método UOP 375-07: Cálculo del Factor de Caracterización UOP y Estimación del Peso Molecular de los Crudos, el cual es el mismo factor de caracterización de Watson , la correlación generalizada de Whitson y el método estándar para el cálculo de la constante viscosidad - Gravedad (VGC), asegurando unos resultados más concretos y fiables al tener en cuenta las distintas variables como lo son: temperatura de ebullición cubica, viscosidad del crudo y la gravedad especifica de las fracciones $\mathrm{C} 7+$ de las muestras analizadas.

A partir de los datos arrojados por los tres métodos evaluados, se encontró que la caracterización según su base de las muestras de crudo de la Subcuenca de Neiva es la siguiente: las muestras de los campos A, y B, son base mixta: NafténicaParafinica, con influencia predominante nafténica; las muestras de los campos C, Dy E, son base mixta: Parafinica- Nafténica, con influencia predominante parafinica y la muestra del campo F: Nafténica-Aromática con influencia predominante nafténica.

A pesar que la temperatura de ebullición, se ve influenciada por agentes desemulsificantes orgámicos de origen aromático, otros métodos que contienen variables distintas a la temperatura se muestran producentes para tener datos precisos y corroborar la exactitud y confiabilidad de utilizar la metodología de destilación con el fin de caracterizar crudos segín su base.

Los datos encontrados en el presente estudio coinciden y complementan los análisis realizados sobre destilación a crudos de las Subcuenca de Neiva.

\begin{tabular}{|c|c|}
\hline Nomenclatura & \\
\hline AyB & Constantes, segín la presión (Para $722 \mathrm{~mm}-\mathrm{Hg}: \mathrm{A}=0.6134$ y B=0.1932) \\
\hline K_W & Factor de caracterización de Watson \\
\hline L & Porcentaje de volumen de Pérdida conregida, \%. \\
\hline L_c & Porcentaje de volumen de pérdida calculado en la prueba, $\%$ \\
\hline $\mathrm{L} \_\mathrm{i}$ & Número de lectura (1, 2, 3, 4, etc.). \\
\hline L_T & Lecturas totales \\
\hline M & Peso molecular de la fracción $\mathrm{C} 7+$ \\
\hline $\mathbf{P}$ & Presión de prueba, $\mathrm{kPa}$ \\
\hline sTc & Temperatura Seudocrítica, $\mathrm{R}$ \\
\hline sPC & Presión Seudocrítica, Psia \\
\hline$T_{7 \theta 0}$ & Temperatura a $1 \mathrm{~atm},{ }^{\circ} \mathrm{C}$ \\
\hline $\mathrm{T}_{\mathrm{b}}$ & Temperatura de ebullición promedia cúbica, $R$ \\
\hline $\mathrm{T}_{\mathrm{p}}$ & Temperatura de prueba, ${ }^{\circ} \mathrm{C}$ \\
\hline TPW & Temperatura Promedio Peso, ${ }^{\circ} \mathrm{F}$ \\
\hline TPM & Temperatura Promedio Molal, ${ }^{\circ} \mathrm{F}$ \\
\hline TPMe & Temperatura Promedio Medio, ${ }^{\circ} \mathrm{F}$ \\
\hline $\mathrm{V}$ & Viscosidad cinemática a $40^{\circ} \mathrm{C}$, (cst) \\
\hline $\mathrm{V} c i$ & Volumen corregido por pérdidas \\
\hline $\mathbf{V}_{i}$ & Volumen destilado en cada lectura \\
\hline$\Delta \mathrm{T}_{\text {Corr }}$ & Corrección que se añade a $\mathrm{T}$ por craqueo térmico, ${ }^{\circ} \mathrm{F}$ \\
\hline$\gamma$ & Gravedad especifica estándar \\
\hline
\end{tabular}

\section{Referencias Bibliográficas}

1. Ahmed, T., 2007. Equations of State and PVT Analysis: Applications for Improved Reservoir Modeling. Gulf Publishing Company, Houston, Texas. 63-99 p.

2. ASTM International, 2004. Standard Test Method for Distillation of Petroleum Products at Atmospheric Pressure, Designation: D86 - 04, West Conshohocken. 22 p. 
3. ASTM International, 2005. Standard Test Method for Calculation of Viscosity-Gravity Constant (VGC) of Petroleum Products, Designation: D2501 - 91, West Conshohocken 3p.

4. Carrillo, J.1985. Propiedades del Petróleo. Universidad Nacional de Colombia. Medellín, Colombia. 61-73 p.

5. Cerutti, A, 2002. La Refinación del petróleo, Tomo I, Ed. Instituto Argentino del Petróleo y del Gas, Argentina. $128 \mathrm{p}$.

6. Gerald L., 2009. Some Aspects of Petroleum Refinery Simulation. En: Speakers Series, Universidad Columbia Británica. $22 \mathrm{p}$.

7. Parra, R, 1997. Diseño, Construcción, Montaje y Puesta en marcha de un equipo de destilación de hidrocarburos liquidos, Universidad Surcolombiana, Neiva. 18-28 p.

8. Perry, R.H. \& Green, D.W. 1997. Perry's Chemical Engineers' Handbook. Seventh Edition McGraw-Hill. EE.UU. $87-88 \mathrm{p}$.

9. Rand, Salvatore J.; 2003. Significance of Tests for Petroleum Products, ASTM Intemational. Seventh Edition. Bridgeport, New Jersey. 258 p.

10. Speight J.G, 2002. Handbook of Petroleum Product Analysis. Honeywell Company. Edit., John Willey Sons, Inc., New Jersey. $140-146$ p.

11. Young, Sydney, 1922. Distillation Principles And Processes, Macmillan \&Co., Limited, ST. Martin's Street, London. 509 p. 